\title{
ON MEAN DISTORTION FOR ANALYTIC FUNCTIONS WITH POSITIVE REAL PART IN A CIRCLE
}

\author{
YÛSAKU KOMATU \\ Dedicated to Professor K. Noshiro on his sixtieth birthday
}

\section{Introduction}

Let $\Re$ be the class of analytic functions $\Phi(z)$ which are regular and of positive real part in the unit circle $|z|<1$ and normalized by $\emptyset(0)=1$. Several distortion theorems have been obtained on various functionals in this class. In a previous paper [4] we have dealt with mean distortion which generalizes a classical theorem of Rogosinski [6].

On the other hand, it is well known that the fundamental operations, integration and differentiation, in ordinary calculus can be analytically interpolated to those of any real order. It has been shown in [5] that such fractional calculus can be used in order to generalize the classical results on angular derivative of Julia [3] and Wolff [7] or Carathéodory [1].

In the present paper we shall show that the notion of fractional calculus is also useful in dealing with distortion theorems in the class $\Re$. Though the results which will be derived below are essentially involved in general theorems obtained in [4], they may be regarded as an interpolating generalization of some illustrating theorems given there. At any rate it will be of some interest to point out concrete cases where the estimates in distortion inequalities are expressed in terms of integrals of elementary functions.

\section{Preliminaries}

Since the unit circle is a convex domain, the fractional integration and differentiation can be defined uniquely for any analytic function regular in the domain as explained in [5] with respect to any reference point in the domain. We suppose here that the reference point lies always at the origin.

Let now $q$ be any positive real number and $\mathscr{L}^{-a}$ denote the integration of

Received July 1, 1966. 
order $q$. Then the operator $\mathscr{L} \equiv \mathscr{L}_{z}$ defined by $\mathscr{L}=z^{-q} \mathscr{D}^{-q}$ is linear and homogeneous of order zero, that is, for any constants $a$ and $b$ the function $\mathscr{L}[a \Phi(z)]$ coincides after substitution $z \mid b z$ with $a \mathscr{L}[\Phi(b z)]$. In fact, we have by definition

$$
\mathscr{D}^{-q} \mathscr{O}(z)=\frac{1}{\Gamma(q)} \int_{0}^{z}(\zeta-z)^{q-1} \mathscr{\emptyset}(\zeta) d \zeta
$$

where the branch of $(z-\zeta)^{q-1} \equiv \exp ((q-1) \log (z-\zeta))$ is determined by taking the principal value of the logarithm and the integration is supposed to be taken along the rectilinear segment from 0 to $z$. Putting $\zeta=t z$, we get

$$
\mathscr{D}^{-q} \mathscr{D}(z)=\frac{z^{q}}{\Gamma(q)} \int_{0}^{1}(1-t)^{q-1} \Phi(t z) d t .
$$

Hence it follows

$$
\begin{aligned}
{\left[Z^{-q} \mathscr{D}_{z}^{-a} a \Phi(Z)\right]^{z=b z} } & =\frac{a}{\Gamma(q)} \int_{0}^{1}(1-t)^{q-1} \Phi(t b z) d t \\
& =a z^{-q} \mathscr{D}^{-a} \Phi(b z)
\end{aligned}
$$

Let next $p$ be any positive real number and $\mathscr{D}^{p}$ denote the differentiation of order $p$. Then the operator $\mathscr{L}=z^{p} \mathscr{D}^{p}$ is also linear and homogeneous of order zero. In fact, let $n=-[-p]$ be the least integer not less than $p$ and $s=n-p ; 0 \leqq s<1$. Then we have by definition

$$
\mathscr{D}^{p}=\mathscr{D}^{n} \mathscr{D}^{-s}, \quad \mathscr{D}^{n} \equiv \frac{d^{n}}{d z^{n}} .
$$

Consequently, it follows

$$
\begin{aligned}
{\left[Z^{p} \mathscr{D}_{z}^{p} a \emptyset(Z)\right]^{Z=b z} } & =\left[Z^{p} \mathscr{D}_{z}^{n} \mathscr{D}_{z}^{-s} a \mathscr{D}(Z)\right]^{z=b z} \\
& =a(b z)^{p} b^{-n} \mathscr{D}_{z}^{n}(b z)^{s} z^{-s} \mathscr{D}_{z}^{-s} \Phi(b z) \\
& =a b^{p-n+s} z^{p} \mathscr{D}_{z}^{n} \mathscr{D}_{z}^{-s} \Phi(b z)=a z^{p} \mathscr{D}_{z}^{p} \Phi(b z) .
\end{aligned}
$$

Since $\mathscr{D}^{0} \equiv \mathscr{D}^{-0}$ is interpreted as the identity, the last relation remains valid obviously in case where $p$ is an integer.

Now, on the other hand, the linear function

$$
\emptyset_{0}(z)=\frac{1+z}{1-z}
$$

mapping $|z|<1$ univalently onto the right-half plane belongs evidently to $\Re$. This function is often distinguished as an extremal function in various variational 
problems within $\Re$. It is well known that the class $\Re$ has a structure formula due to Herglotz [2] which may be stated as follows:

It is necessary and sufficient for $\Phi \in \mathfrak{R}$ that $\Phi$ is representable by means of Herglotz integral

$$
\Phi(z)=\int_{-\pi}^{\pi} \Phi_{0}\left(e^{-i \varphi} z\right) d \mu(\varphi)
$$

where $\rho(\varphi)$ is a real-valued function defined for $-\pi<\varphi \leqq \pi$ which is increasing and has the total variation equal to unity.

\section{Mean distortion of fractional integral}

We are now in position to formulate our main result. We begin with a mean distortion for fractional integral of $\emptyset \in \mathfrak{R}$.

THEOREM 1. Let $q>0$ and $\lambda \geqq 1$ be any real numbers. Then, for any $\emptyset \in \mathfrak{R}$ and $0 \leqq r<1$, we have

$$
\int_{-\pi}^{\pi}\left|\mathscr{D}^{-q} \emptyset\left(r e^{i \theta}\right)\right|^{\lambda} d \theta \leqq \frac{r^{a \lambda}}{\Gamma(q)^{\lambda}} \int_{-\pi}^{\pi}\left|\int_{0}^{1}(1-t)^{q-1} \frac{1+t r e^{i \theta}}{1-t r e^{i \theta}} d t\right|^{\lambda} d \theta
$$

For any fixed $r$ with $0<r<1$, the equality sign holds if and only if $\Phi(z)$ is of the form $\emptyset_{0}(\varepsilon z) \equiv(1+\varepsilon z) /(1-\varepsilon z)$ with $|\varepsilon|=1$.

Proof. By making use of Herglotz representation of $\emptyset$, we get

$$
\begin{aligned}
\mathscr{D}^{-q} \emptyset(z) & =\frac{z^{q}}{\Gamma(q)} \int_{0}^{1}(1-t)^{q-1} \emptyset(t z) d t \\
& =\frac{z^{q}}{\Gamma(q)} \int_{0}^{1}(1-t)^{q-1} d t \int_{-\pi}^{\pi} \emptyset_{0}\left(e^{-i \varphi} t z\right) d \rho(\varphi)
\end{aligned}
$$

and hence

$$
\int_{-\pi}^{\pi}\left|\mathscr{D}^{-q} \Phi\left(r e^{i \theta}\right)\right|^{\lambda} d \theta=\frac{r^{q \lambda}}{\Gamma(q)^{\lambda}} \int_{-\pi}^{\pi} d \theta\left|\int_{-\pi}^{\pi} d \rho(\varphi) \int_{0}^{1}(1-t)^{q-1} \emptyset_{0}\left(t r e^{i(\theta-\phi)}\right) d t\right|^{\lambda} .
$$

Since $\xi^{\lambda}$ is an increasing convex function for $\xi \geqq 0$ and $\rho(\varphi)$ is an increasing function with total variation equal to unity, the last relation yields

$$
\begin{aligned}
\int_{-\pi}^{\pi}\left|\mathscr{D}^{-q} \mathscr{D}\left(r e^{i \theta}\right)\right|^{\lambda} d \theta & \leqq \frac{r^{q \lambda}}{\Gamma(q)^{\lambda}} \int_{-\pi}^{\pi} d \theta \int_{-\pi}^{\pi} d o(\varphi)\left|\int_{0}^{1}(1-t)^{q-1} \Phi_{0}\left(t r e^{i(\theta-p)}\right) d t\right|^{\lambda} \\
& =\frac{r^{q \lambda}}{\Gamma(q)^{\lambda}} \int_{-\pi}^{\pi}\left|\int_{0}^{1}(1-t)^{q-1} \Phi_{0}\left(t r e^{i \theta}\right) d t\right|^{\lambda} d \theta
\end{aligned}
$$

This is the desired inequality. 
Concerning the equality sign we first consider the case $\lambda=1$. Then any function $\rho(\varphi)$ associated with an extremal function $\emptyset$ is characterized by the condition

$$
\begin{aligned}
& \left|\int_{-\pi}^{\pi} d \rho(\varphi) \int_{0}^{1}(1-t)^{q-1} \emptyset_{0}\left(t r e^{i(\theta-\varphi)}\right) d t\right| \\
= & \int_{-\pi}^{\pi} d \rho(\varphi)\left|\int_{0}^{1}(1-t)^{q-1} \emptyset_{0}\left(t r e^{i(\theta-\varphi)}\right) d t\right|
\end{aligned}
$$

valid for every $\theta$ from $-\pi<\theta \leqq \pi$, since its both members are continuous in $\theta$. Define the function

$$
\Psi(z)=\int_{0}^{1}(1-t)^{q-1} \emptyset_{0}(t z) d t\left(\equiv \Gamma(q) z^{-q} \mathscr{D}^{-q} \mathscr{D}_{0}(z)\right)
$$

which is evidently regular and of positive real part for $|z| \leqq r<1$. The above condition is then equivalent to the requirement that for every value of $\theta$ the quantity $\Psi\left(r e^{i(\theta-\varphi)}\right)$ possesses the same argument for all $\varphi$ with $d \rho(\varphi)>0$ over $-\pi<\varphi \leqq \pi$. But it is shown that this quantity can never have the same argument for any distinct values of $\varphi$. In fact, suppose that $\Psi\left(z e^{-i \varphi}\right)$ has the same argument at $\varphi_{0}$ and $\varphi_{1}$. Then the function defined by

$$
X(z)=\frac{\Psi\left(z e^{-i \varphi_{1}}\right)}{\Psi\left(z e^{-i \varphi_{0}}\right)}
$$

is regular throughout $|z| \leqq r$ and remains real along the circumference $|z|=r$. Hence it must reduce to a constant, i.e. $X(z) \equiv X(0)=1$. Since it holds

$$
\left[\frac{d}{d z} \Psi\left(z e^{-i \varphi}\right)\right]^{2=0}=e^{-i \phi} \Psi^{\prime}(0)=\frac{2}{q(q+1)} e^{-i \varphi}
$$

$\varphi_{0}$ and $\varphi_{1}$ must coincide. Consequently, $\rho(\varphi)$ remains constant except at a single jump with the height necessarily equal to unity. Thus it is shown that the form of extremal functions is given by $\emptyset_{0}(\varepsilon z)$ with $|\varepsilon|=1$. Finally, in case $\lambda>1$, i.e. the case where $\xi^{\lambda}$ is strictly convex, it is evident that the above condition is necessary. It is also directly obvious that the condition is sufficient.

The statement in theorem 1 remains true for $q=0$, provided the estimate in the right-hand member is understood to be replaced by its limit as $q \rightarrow+0$. It reduces then trivially to

$$
\lim _{l \rightarrow+0} \frac{r^{q \lambda}}{\Gamma(q)^{\lambda}} \int_{-\pi}^{\pi}\left|\int_{0}^{1}(1-t)^{q-1} \frac{1+t r e^{i \theta}}{1-t r e^{i \theta}} d t\right|^{\lambda} d \theta=\int_{-\pi}^{\pi}\left|\frac{1+r e^{i \theta}}{1-r e^{i \theta}}\right|^{\lambda} d \theta .
$$


We remark that the particular case $\lambda=2$ of theorem 1 can be verified alternatively in a simple way. In fact, let the Taylor expansion of $\boldsymbol{\emptyset}(z)$ be

$$
\Phi(z)=1+\sum_{\nu=1}^{\infty} c_{\nu} z^{\nu}, \quad c_{\nu}=2 \int_{-\pi}^{\pi} e^{-i \nu \varphi} d \rho(\varphi) \quad(\nu=1,2, \ldots) .
$$

Since the operation $\mathscr{D}^{-a}$ is applicable termwise, we get

$$
\begin{gathered}
\mathscr{D}^{-q} \emptyset(z)=\frac{1}{\Gamma(1+q)} z^{q}+\sum_{\nu=1}^{\infty} c_{\nu} \frac{\nu !}{\Gamma(1+\nu+q)} z^{\nu+q}, \\
\frac{1}{2 \pi} \int_{-\pi}^{\pi}\left|\mathscr{D}^{-q} \emptyset\left(r e^{i \theta}\right)\right|^{2} d \theta=\frac{1}{\Gamma(1+q)^{2}} r^{2 q}+\sum_{\nu=1}^{\infty}\left|c_{\nu}\right|^{2} \frac{\nu !^{2}}{\Gamma(1+\nu+q)^{2}} r^{2(\nu+q)} .
\end{gathered}
$$

In view of $\left|c_{\nu}\right| \leqq 2(\nu \geqq 1)$ there follows readily

$$
\frac{1}{2 \pi} \int_{-\pi}^{\pi}\left|\mathscr{D}^{-q} \Phi\left(r e^{i \theta}\right)\right|^{2} d \theta \leqq \frac{1}{\Gamma(1+q)^{2}} r^{2 q}+4 \sum_{\nu=1}^{\infty} \frac{\nu !^{2}}{\Gamma(1+\nu+q)^{2}} r^{2(\nu+q)},
$$

which is equivalent to the desired inequality. The assertion on extremal functions is immediate.

\section{Mean distortion of fractional derivative}

We next give a corresponding mean distortion for fractional derivative of $\emptyset \in \Re$.

THEOREM 2. Let $p>0$ and $\lambda \geqq 1$ be any real numbers and $n=-[-p], p=n-s$. Then, for any $\emptyset \in \Re$ and $0 \leqq r<1$, we have

$$
\begin{aligned}
& \int_{-\pi}^{\pi}\left|\mathscr{D}^{p} \emptyset\left(r e^{i \theta}\right)\right|^{\lambda} d \theta \\
& \leqq \frac{s}{r^{p}} \int_{-\pi}^{\pi} \mid \int_{0}^{1}(1-t)^{s-1}\left(\frac{1}{\Gamma(1-p)} \frac{1+t r e^{i \theta}}{1-t r e^{i \theta}}\right. \\
& \left.\quad+2 \sum_{j=1}^{n} \frac{n !}{(n-j) !} \frac{1}{\Gamma(1+j-p)} \frac{\left(t r e^{i \theta}\right)^{j}}{\left(1-t r e^{i \theta}\right)^{j+1}}\right)\left.d t\right|^{\lambda} d \theta .
\end{aligned}
$$

The function $\emptyset_{0}(\varepsilon z)$ with $|\varepsilon|=1$ is always an extremal function for this estimation.

Proof. Herglotz representation yields

$$
\mathscr{D}^{p} \mathscr{\Phi}(z)=\int_{-\pi}^{\pi} \mathscr{D}^{p} \Phi_{0}\left(e^{-i \varphi} z\right) d \rho(\varphi),
$$

and hence

$$
\int_{-\pi}^{\pi}\left|\mathscr{V}^{p} \Phi\left(r e^{i \theta}\right)\right|^{\lambda} d \theta=\int_{-\pi}^{\pi} d \theta\left|\int_{-\pi}^{\pi} \mathscr{S}^{p} \Phi_{0}\left(e^{-i \varphi} r e^{i \theta}\right) d \rho(\varphi)\right|^{\lambda}
$$




$$
\leqq \int_{-\pi}^{\pi} d \theta \int_{-\pi}^{\pi}\left|\mathscr{P}^{p} \Phi_{0}\left(r e^{i(\theta-?)}\right)\right|^{\lambda} d \rho(\varphi)=\int_{-\pi}^{\pi}\left|\mathscr{D}^{p} \Phi_{0}\left(r e^{i \theta}\right)\right|^{\lambda} d \theta
$$

It remains only to compute the last estimate in explicit form. Based on the definition of fractional derivative, we have

$$
\begin{aligned}
\mathscr{D}^{p} \varpi_{0}(z) & =\mathscr{D}^{n} \frac{z^{s}}{\Gamma(s)} \int_{0}^{1}(1-t)^{s-1} \emptyset_{0}(t z) d t \\
& =s \sum_{j=0}^{n}\left(\begin{array}{l}
n \\
j
\end{array}\right) \frac{z^{s-n+j}}{\Gamma(s-n+j+1)} \int_{0}^{1}(1-t)^{s-1} t^{j} \emptyset_{0}(t z) d t \\
& =s \int_{0}^{1}(1-t)^{s-1}\left(\frac{z^{-p}}{\Gamma(1-p)} \emptyset_{0}(t z)+\sum_{j=1}^{n} \frac{n ! t^{j} z^{-p+j}}{(n-j) ! j ! \Gamma(1+j-p)} \emptyset_{0}^{(j)}(t z)\right) d t .
\end{aligned}
$$

Thus, by remembering

$$
\Phi_{0}(t z)=\frac{1+t z}{1-t z}, \quad \Phi_{0}^{(j)}(t z)=\frac{j ! 2}{(1-t z)^{j+1}} \quad(j=1,2, \ldots),
$$

the desired form follows immediately by direct substitution.

If, in particular, $p$ is equal to a positive integer $n$, the estimate in the righthand member in theorem 2 becomes briefly

$$
\lim _{p \rightarrow n-0} \int_{-\pi}^{\pi}\left|\mathscr{D}^{p} \Phi_{0}\left(r e^{i \theta}\right)\right|^{\lambda} d \theta=\int_{-\pi}^{\pi}\left|\mathscr{D}^{n} \emptyset_{0}\left(r e^{i \theta}\right)\right|^{\lambda} d \theta=(n ! 2)^{\lambda} 2 \int_{0}^{\pi} \frac{d \theta}{\left|1-r e^{i \theta}\right|^{(n+1) \lambda}} .
$$

This is a case previously dealt with in [4].

Finally we supplement a remark on the characterization of extremal functions in theorem 2, though it will be not quite complete. Any function $\Phi_{0}(s z)$ with $|\varepsilon|=1$ is surely an extremal function as mentioned in the theorem. Now, it depends only on the behavior of the elementary function $\emptyset_{0}(z)$ whether there exists besides an extremal function or not. In fact, in case $\lambda=1$, any function $\rho(\varphi)$ associated with an extremal function $\Phi$ is characterized by the relation

$$
\left|\int_{-\pi}^{\pi} \mathscr{D}^{p} \Phi_{0}\left(r e^{i(\theta-) \varphi}\right) d \rho(\varphi)\right|=\int_{-\pi}^{\pi}\left|\mathscr{D}^{p} \Phi_{0}\left(r e^{i(\theta-\varphi)}\right)\right| d \rho(\varphi)
$$

valid for every $\theta$ from $-\pi<\theta \leqq \pi$, since its both members are continuous in $\theta$. This condition is equivalent to the requirement that for every $\theta$ the quantity $\mathscr{D}^{p} \emptyset_{0}\left(r e^{i(\theta-\rho)}\right)$ possesses the same argument for all $\varphi$ with $d \rho(\varphi)>0$ over $-\pi<\varphi \leqq \pi$.

Now, if it could be shown that $\mathscr{D}^{p} \Phi_{0}(z)$ does not vanish for $0<|z| \leqq r$, we can prove that the quantity $\mathscr{D}^{p} \Phi_{0}\left(r e^{i(\theta-\varphi)}\right)$ has never the same argument for 
any distinct values of $\varphi$. In fact, the function $z^{p} \mathscr{D}^{p} \mathscr{D}_{0}(z)$ is regular for $|z| \leqq r$. Suppose that $z^{p} \mathscr{Q}^{p} \mathscr{D}_{0}\left(z e^{-i ;}\right)$ has the same argument at $\varphi_{0}$ and $\varphi_{1}$. Then the quotient

$$
Y(z)=\frac{\mathscr{E}^{p} \Phi_{0}\left(z e^{-i \varphi_{0}}\right)}{\mathscr{D}^{p} \Phi_{0}\left(z e^{-i \varphi_{1}}\right)}
$$

is regular throughout $|z| \leqq r$ and remains real along $|z|=r$. Hence it must reduce to a constant, i.e. $Y(z) \equiv Y(0)=1$. Consequently, in view of

$$
\left[\frac{d}{d z} z^{p} \mathscr{D}^{p} \Phi_{0}\left(z e^{-i \rho}\right)\right]^{z=0}=\frac{2 e^{-i \varphi}}{\Gamma(2-p)}
$$

we conclude that $\varphi_{0}$ and $\varphi_{1}$ cannot differ provided $p \neq 2,3, \ldots$ But if $p$ is a positive integer, then we get

$$
\mathscr{D}^{p} \Phi_{0}\left(z e^{-i \varphi}\right)=\frac{p ! 2 e^{-i \not \rho}}{\left(1-z e^{-i \varphi}\right)^{p+1}}
$$

and hence $\varphi_{0}$ and $\varphi_{1}$ cannot differ surely.

Consequently, it is verified that extremal functions in theorem 2 for $\lambda=1$ and a fortiori for $\lambda>1$ must be of the form $\Phi_{0}(\varepsilon z)$ with $|\varepsilon|=1$, provided that $\mathscr{Q}^{p} \Phi_{0}(z)$ does not vanish for $0<|z| \leqq r$. Further it has been shown that the last-mentioned condition on $\mathscr{V}^{p} \Phi_{0}(z)$ is really satisfied for any positive integer $p$.

It may be noted that, for any $p>0$ the condition $\mathscr{V}^{p} \Phi_{0}(z) \neq 0$ for $0<|z| \leqq r$ is naturally fulfilled provided $r$ is sufficiently close to zero.

On the other hand, the particular case $\lambda=2$ can be dealt with similarly as before. In fact, putting

$$
\emptyset(z)=1+\sum_{\nu=1}^{\infty} c_{\nu} z^{\nu}, \quad c_{\nu}=2 \int_{-\pi}^{\pi} e^{-i \nu \rho} d \rho(\varphi) \quad(\nu=1,2, \ldots),
$$

we get

$$
\begin{gathered}
\mathscr{D}^{p} \mathscr{D}(z)=\frac{1}{\Gamma(1-p)} z^{-p}+\sum_{\nu=1}^{\infty} c_{\nu} \frac{\nu !}{\Gamma(1+\nu-p)} z^{\nu-p}, \\
\frac{1}{2 \pi} \int_{-\pi}^{\pi}\left|\mathscr{D}^{p} \mathscr{\Phi}\left(r e^{i \theta}\right)\right|^{2} d \theta=\frac{1}{\Gamma(1-p)^{2}} r^{-2 p}+\sum_{\nu=1}^{\infty}\left|c_{\nu}\right|^{2} \frac{\nu !^{2}}{\Gamma(1+\nu-p)^{2}} r^{2(\nu-p)} .
\end{gathered}
$$

Consequently, we obtain the mean distortion in the form

$$
\frac{1}{2 \pi} \int_{-\pi}^{\pi}\left|\mathscr{D}^{p} \emptyset\left(r e^{i p}\right)\right|^{2} d \theta \leqq \frac{1}{\Gamma(1-p)^{2}} r^{-2 p}+4 \sum_{\nu=1}^{\infty} \frac{\nu !^{2}}{\Gamma(1+\nu-p)^{2}} r^{2(\nu-p)} .
$$


The extremal functions in this case are only those of the form $\Phi_{0}(\varepsilon z)$ with $|\varepsilon|=1$, because even a single relation $\left|c_{\nu}\right|=2$ for some $\nu$ implies $\Phi(z)=\emptyset_{0}(\varepsilon z)$ with $\varepsilon^{\nu}=c_{\nu} / 2$.

\section{REFERENCES}

[1] Carathéodory, C.: Über die Winkelderivierten von beschränkten analytischen Funktionen, Sitzungsber. Preuss. Akad. (1929), 1-18.

[2] Herglotz, A.: Über Potenzreihen mit positivem, reellem Teil im Einheitskreise, Leipziger Ber., 63 (1911), 501-511.

[3] Julia. G.: Extensions nouvelles d'un lemme de Schwarz, Acta Math., 42 (1920), 349-355.

[4] Komatu, Y.: On analytic functions with positive real part in a circle, Kōdai Math. Sem. Rep., 10 (1958), 64-83.

[5] Komatu, Y.: On fractional angular derivative, Kōdai Math. Sem. Rep., 13 (1961), 249-254.

[6] Rogosinski, W.: Über positive harmonische Entwicklungen und typisch-reelle Potenzreihen, Math. Zeitschr., 35 (1932), 93-121.

[7] Wolff, J.: Sur une généralisation d'un théorème de Schwarz, C. R. Paris, 183 (1926), 500-502.

Tokyo Institute of Technology 